\title{
Desafíos de la investigación en tiempos de pandemia
}

Research challenges in times of pandemic

\author{
Subcomisión de Ética Clínica y Subcomisión de Investigación Pediátrica
}

http:/ / dx.doi.org/10.5546/ aap.2021.S36

Cómo citar: Subcomisión de Ética Clínica Subcomisión de Investigación Pediátrica. Desafíos de la investigación en tiempos de pandemia. Arch Argent Pediatr 2021;119(1):S36-S38.

Las emergencias sanitarias globales, como lo es la pandemia por coronavirus tipo 2 del síndrome respiratorio agudo grave (severe acute respiratory syndrome coronavirus 2; $S A R S-C o V-2$, por sus siglas en inglés), suponen escenarios complejos que implican un desafío para la salud pública. A las necesidades de organización de los recursos disponibles, se les suma la urgencia y la incertidumbre frente a un virus de alta contagiosidad del cual nada se sabía y que se va aprendiendo sobre la marcha.

Al mismo tiempo, el miedo generado en la población mundial por la realidad vivida en cada país afectado, así como el exceso de información, generan presión desde la sociedad para que la medicina ofrezca una rápida respuesta a esta agresión, pero se desconocen los tiempos necesarios para poder producir un avance científico de eficacia y seguridad comprobadas. Desde el comienzo de la pandemia, se utilizaron, sin suficiente evidencia,

Correspondencia: Dr. Santiago Vidaurreta: svidaurreta@gmail.com

Financiamiento:

Ninguno.

Conflicto de intereses: Ninguno que declarar.

Recibido: 15-9-2020 Aceptado: 22-9-2020 tratamientos eficaces en otras patologías (hidroxicloroquina, azitromicina, antirretrovirales, anticuerpos monoclonales, etc.). ${ }^{1}$ Simultáneamente, y con el fin de demostrar la eficacia, seguridad y efectividad de la medicación utilizada, se pusieron en marcha diferentes investigaciones y comenzó el desarrollo de los estudios relacionados con la obtención de una vacuna. A modo de ejemplo, una consulta realizada el 8 de septiembre de 2020 en el sitio clinicaltrials.gov, que incluyó los términos COVID-19 y SARSCoV-2, arrojó 3402 investigaciones registradas. ${ }^{2}$ También llama la atención que, si bien la enfermedad tiene un impacto mucho más significativo en la población adulta, 500 de esos estudios corresponden a la población pediátrica (menores de 18 años).

En la búsqueda de una respuesta a la enfermedad y frente a presiones sociales, políticas y económicas, existe un riesgo cierto de que se vean afectados el marco ético y científico que rige las investigaciones con seres humanos. Este riesgo se acentúa aún más cuando las investigaciones son realizadas en poblaciones vulnerables, ya sea por condiciones sociales o por edad, como sucede en pediatría. Es por eso por lo que la respuesta de la ciencia ante la emergencia no debe soslayar el rigor científico ni las pautas éticas imprescindibles para llevarla a cabo $\mathrm{y}$, en estas excepcionales condiciones, es cuando más se deben considerar los resguardos éticos que regulan las investigaciones y que dan protección a aquellos que participan de estas.

Desde una visión bioética, se encuentran involucrados todos los principios. La búsqueda de un beneficio que evite producir daño, el respeto por la autonomía de los participantes y la distribución equitativa de los beneficios y cargas de la investigación son principios elementales que debieran ser siempre respetados a fin de preservar la dignidad que merecen todos aquellos que decidieron participar como sujetos de investigación. 
Se deberá considerar que "aunque el objetivo principal de la investigación médica es generar nuevos conocimientos, nunca debe tener primacía sobre los derechos y los intereses de la persona que participa en la investigación". ${ }^{3}$ De allí que, independientemente de las urgencias y del interés de lograr resultados positivos rápidos, la ciencia no debería estar por "encima" de los intereses y protección de los participantes de los estudios.

En guías y recomendaciones ${ }^{4,5}$ relacionadas con el marco ético que deben guardar las investigaciones en épocas de epidemias o pandemias, en general, no se hace referencia a las investigaciones por realizarse en la población pediátrica. Sin embargo, algunos consideran que, en investigaciones realizadas en emergencias sanitarias globales, "no deberían aplicarse criterios de exclusión automática de los 'grupos vulnerables', tales como niños, mujeres, embarazadas o personas mayores. En la práctica, la exclusión puede hacer que esos grupos se tornen aún más vulnerables". ${ }^{6}$

En este contexto, las Subcomisiones de Ética Clínica y de Investigación Pediátrica de la Sociedad Argentina de Pediatría se han reunido para reflexionar sobre los aspectos mencionados. Es evidente que la situación obliga a que todos los actores colaboren para permitir que la ciencia busque y obtenga las respuestas en el menor tiempo posible. Esto incluye, fundamentalmente, mecanismos administrativos expeditivos. Pero, de ninguna manera, debe confundirse una ventaja tal con la flexibilización del rigor científico y ético con que cada proyecto deba ser examinado.

A su vez, si bien debería darse prioridad a las investigaciones relacionadas con la emergencia actual, la preocupación por investigar sobre la pandemia no debería dejar de lado, dentro de lo posible, aquellas investigaciones en curso que podrían contribuir a un beneficio para otras patologías que requieren también de desarrollos para su tratamiento o prevención en la infancia.

Además, la investigación no debe agotarse en la respuesta terapéutica a una enfermedad con limitado compromiso clínico en la infancia, sino que debe extenderse al impacto indirecto que produce en esta población, que ya se muestra muy significativo. ${ }^{7}$ Aceptando lo expresado en cuanto al menor impacto de la pandemia de COVID-19 en pediatría, existen situaciones relacionadas con ella, tales como la posibilidad de trasmisión vertical o durante la lactancia, la aparición de nuevas manifestaciones, como el síndrome inflamatorio multisistémico, la adecuación de tratamientos utilizados en adultos en que se compruebe su seguridad y eficacia, así como las dosis, formulación, efectividad y seguridad de las posibles vacunas, que avalan la realización de estudios en niños por ser situaciones propias de la edad pediátrica.

Una mención especial requiere la mesura con que los medios de difusión deberían abordar el tema para evitar generar ansiedad innecesaria y falsas expectativas en una población de por sí profundamente preocupada. Sería necesario realizar una evaluación crítica de la información ágil y eficaz por parte de los científicos, junto con una adecuada estrategia de comunicación de periodistas o influencers para incorporar a la sociedad a una genuina discusión sobre temas científicos. ${ }^{8}$ En este sentido, la responsabilidad es aún mayor cuando se refiere a profesionales que dirigen investigaciones. No debe olvidarse que la difusión de estudios clínicos con fines de reclutamiento a través de la prensa u otros medios debe contar con la aprobación de un comité de ética y debe llevarse a cabo en los términos exactos en que se autorizó. ${ }^{9}$

La Sociedad Argentina de Pediatría remarca la importancia de fomentar y promover la investigación en pediatría, ya que no hacerlo priva a los niños de acceder a los avances científicos con evidencia comprobada. ${ }^{10}$ Esta premisa también debe sostenerse en el caso de esta nueva enfermedad y evitar, así, que, una vez más, los niños queden excluidos de las investigaciones que pueden aportarles tratamientos seguros y eficaces. ${ }^{11}$

\section{REFERENCIAS}

1. Área de Farmacia, Hospital Garrahan. Recomendaciones de organismos, agencias de salud, y sociedades científicas nacionales e internacionales para el tratamiento farmacológico de Covid 19. Versión 3. Julio de 2020 [Consulta: 3 de septiembre 2020]. Disponible https:// www.garrahan.gov.ar/PDFS/medicamentos/ROAST_ COVID-19.pdf.

2. ClinicalTrials.gov [Página de Internet]. Bethesda: National Library of Medicine. [Consulta: 8 de septiembre 2020]. Disponible en: https: / / clinicaltrials.gov/.

3. Declaración de Helsinki de la AMM-Principios éticos para las investigaciones médicas en seres humanos. [Consulta: 8 de septiembre 2020]. Disponible en: http: / / www.anmat. gov.ar/comunicados/HELSINSKI_2013.pdf.

4. Programa Regional de Bioética, Departamento de Sistemas y Servicios de Salud. Orientación ética sobre cuestiones planteadas por la pandemia del nuevo coronavirus (COVID-19). Washington, DC: Organización Panamericana de la Salud, 2020. [Consulta: 16 de julio de 2020]. Disponible en: https://iris.paho.org/bitstream/ handle/10665.2 / 52142 / OPSHSSBIOCOVID-19200008_ spa.pdf?sequence $=1$ \&isAllowed $=y$. 
5. Investigación en situaciones de desastre y brotes de enfermedades. En Organización Mundial de la Salud; Consejo de Organizaciones Internacionales de las Ciencias Médicas. Pautaséticas internacionales paralainvestigación relacionada con la salud con seres humanos. $4{ }^{\text {ta }}$ ed. Ginebra:CIOMS; 2016:83-88. [Consulta:16 dejulio de2020]. Disponible en: https:/ / cioms.ch/publications/product/ pautas-eticas-internacionales-para-la-investigacionrelacionada-con-la-salud-con-seres-humanos/.

6. Investigación en emergencias sanitarias globales: cuestiones éticas breve reseña. Nuffield Council on Bioethics, 2020. [Consulta: 21 de julio de 2020]. Disponible en: https:/ / www.nuffieldbioethics.org/assets / pdfs / Research-inglobal-health-emergencies-short-report-ESPANOL.pdf.

7. WHO-UNICEF-LancetCommissioners. AfterCOVID-19, a future for the world's children? Lancet. 2020;396(10247):298300.

8. Casado i Pere Puigdomènech M. Documento sobre los aspectos éticos del diálogo entre ciencia y sociedad. Barcelona: Universitat de Barcelona; 2018.
9. Argentina. Ministerio de Salud. Administración Nacional de Medicamentos, Alimentos y Tecnología Médica. Resolución 1480/2011. Guía para Investigaciones con Seres Humanos. Buenos Aires, 13 de septiembre de 2011. [Consulta: 27 de agosto 2020]. Disponible en: http:/ / www. anmat.gov.ar/webanmat/legislacion/medicamentos/ Resolucion_1480-2011.pdf.

10. Investigador. En Guía Armonizada de ICH anexo Integrado a ICH E6 (R1): Guía para la buena práctica clínica ICH E6 (R2) Pauta de consenso de ICH. [Consulta: 27 de agosto de 2020]. Disponible en: https: / / ichgcp.net/es / 4-investigator.

11. Sociedad Argentina dePediatría. Investigación en Pediatría. [Consulta: 20 de julio de 2020]. Disponible en: https:// www.sap.org.ar/uploads/archivos/files_cumunicadoinvestigaciones-2014_1489959595.pdf.

12. Hwang TJ, Randolph AG, Bourgeois FT. Inclusion of Children in Clinical Trials of Treatments for Coronavirus Disease 2019 (COVID-19). JAMA Pediatr. 2020;174(9):8256. 